\title{
Predictors of quality of life among adolescents and young adults with a bleeding disorder
}

John M. McLaughlin 1*, James E. Munn², Terry L. Anderson', Angela Lambing ${ }^{3,5}$, Bartholomew Tortella and Michelle L. Witkop ${ }^{4}$

\begin{abstract}
Background: Health-related quality of life (HRQoL) in adolescents and young adults with bleeding disorders is under-researched. We aimed to describe factors related to HRQoL in adolescents and young adults with hemophilia A or B or von Willebrand disease.

Methods: A convenience sample of volunteers aged 13 to 25 years with hemophilia or von Willebrand disease completed a cross-sectional survey that assessed Physical (PCS) and Mental (MCS) Component Summary scores on the SF-36 questionnaire. Quantile regression models were used to assess factors associated with HRQOL.
\end{abstract}

Results: Of 108 respondents, 79, 7, and 14\% had hemophilia A, hemophilia B, and von Willebrand disease, respectively. Most had severe disease (71\%), had never developed an inhibitor (65\%), and were treated prophylactically (68\%). Half of patients were aged 13 to 17 years and most were white (80\%) and non-Hispanic (89\%). Chronic pain was reported as moderate to severe by $31 \%$ of respondents. Median PCS and MCS were 81.3 and 75.5, respectively. Quantile regression showed that the median PCS for women (61\% with von Willebrand disease) was 13.1 (95\% Cl: 2.4, 23.8; $p=0.02$ ) points lower than men. Ever developing an inhibitor (vs never) was associated with a 13.1-point (95\% Cl: 4.7, 21.5; $p<0.01)$ PCS reduction. MCS was 10.0 points ( $95 \% \mathrm{Cl}: 0.7,19.3 ; p=0.04$ ) higher for prophylactic infusers versus those using on-demand treatment. Compared with patients with no to mild chronic pain, those with moderate to severe chronic pain had 25.5-point (95\% Cl: 17.2, 33.8; $p<0.001)$ and 10.0-point (95\% Cl: $0.8,19.2 ; p=0.03)$ reductions in median PCS and MCS, respectively.

Conclusions: Efforts should be made to prevent and manage chronic pain, which was strongly related to physical and mental HRQoL, in adolescents and young adults with hemophilia and von Willebrand disease. Previous research suggests that better clotting factor adherence may be associated with less chronic pain.

Keywords: Chronic pain, Hemophilia, Pain management, Patient adherence, Prophylaxis, von Willebrand disease

\footnotetext{
* Correspondence: john.mclaughlin@pfizer.com

${ }^{1}$ Pfizer Inc, Global Innovative Pharma, 500 Arcola Rd, Collegeville, PA 19426,

USA

Full list of author information is available at the end of the article
} 


\section{Background}

Therapeutic advancements [1-4], improved treatment approaches [5-8], and enhanced care delivery models [9-12] have resulted in highly active lifestyles and nearnormal life expectancy for persons diagnosed with a bleeding disorder [13]. As treatments continue to improve, patient care will continue to evolve toward preventing bleeding disorder complications, such as hemophilic arthropathy, chronic pain, and inhibitor development-with the ultimate goal of attaining and maintaining high health-related quality of life (HRQoL) for those affected by the disorder [14]. Previous research has shown that among persons with a bleeding disorder, HRQoL may be related to pain, treatment type (prophylaxis vs on-demand), adherence, age-related concerns, inhibitor status, or depression [14-21]. These findings suggest that bleeding disorders particularly diminish the physical aspects of HRQoL and that alleviating the clinical burden of bleeding disorders may be particularly important for improving and maintaining physical wellbeing and overall HRQoL.

Measuring HRQoL is a unique way to assess the general well-being and perceived health of individuals living with a bleeding disorder, the experiences related to having a bleeding disorder, and satisfaction with treatment [22]. However, very little, if any, data exist that describe HRQoL among adolescent and young adult (AYA) persons diagnosed with a bleeding disorder. AYAs are a unique population of bleeding disorder patients who are often just starting to take more responsibility for the management of their own disease and developing treatment habits that can carry over into adult life [23]. Entering adolescence and young adulthood might mean finally confronting the demands that managing a bleeding disorder entails. The aim of this study was to investigate the relationships between pain management, adherence to prescribed treatment, and HRQoL in AYA persons with hemophilia (PWH) or von Willebrand disease (VWD).

\section{Methods}

\section{Study population and recruitment}

Data describing HRQoL, adherence to prescribed treatment regimens, and level of chronic pain among AYA PWH or VWD were obtained as part of the larger Interrelationship Between Management of Pain, Adherence to Clotting-factor Treatment, and Quality of Life (IMPACT QoL) study. As the name suggests, the IMPACT QoL study had the primary goal of assessing the relationship between validated measures of pain, clotting-factor adherence, and HRQoL among AYA PWH or VWD. Data describing the relationship between adherence to a prescribed clotting factor treatment regimen and chronic pain, along with racial differences in chronic pain and
QoL from this study, were previously reported [24, 25]. Data were collected via a one-time, cross-sectional, online survey from a convenience sample of AYA PWH or VWD. To be eligible to complete the survey, participants had to be aged 13 to 25 years, read, write, and speak English, and have hemophilia A, hemophilia B, or VWD. Recruitment occurred at major US hemophilia meetings (e.g., Inhibitor Summits and National Hemophilia Foundation meetings), US hemophilia treatment centers, and through a Facebook ${ }^{\odot}$ page dedicated to the study from April through December of 2012. All surveys were completed electronically using SurveyMonkey and Apple ${ }^{\circ}$ iPads ${ }^{\circledR}$. Informed consent was obtained from all individual participants included in the study. The study was approved by the Munson Medical Center (Traverse City, MI) institutional review board prior to data collection. All data were de-identified prior to analysis. The current study uses the IMPACT QoL survey data to determine factors associated with better HRQoL among AYA (aged 13-25 years) PWH or VWD.

\section{Measurement}

HRQoL was measured using the 36-Item Short Form Health Survey (SF-36). The SF-36 is composed of 8 multi-item scales (35 items) assessing the following: (1) bodily pain (BP, 2 items), (2) physical function (PF, 10 items), (3) role limitations due to physical health problems (RP, 4 items), (4) general health (GH, 5 items), (5) vitality (VT, 4 items), (6) social functioning (SF, 2 items), and (7) role limitations due to emotional problems (RE, 3 items) and emotional well-being/mental health (MH, 5 items) [26]. The 36th item, which asks about health change, is not included in the scale or summary scores. Each subscale is calculated by taking the average of the participant's responses to the questions contained in the subscale and then standardizing it so that each had a final range of 0 (lowest level of functioning) to 100 (highest level of functioning). These eight scales can be aggregated into two summary measures: the Physical (PCS) and Mental (MCS) Component Summary scores, where higher scores represent better health. The scoring algorithm for PCS includes positive weights for the PF, $\mathrm{RP}, \mathrm{BP}, \mathrm{GH}$, and VT scales and negative weights for the $\mathrm{SF}, \mathrm{RE}$, and $\mathrm{MH}$. The scoring algorithm for MCS includes positive weights for the VT, SF, RE, and $\mathrm{MH}$ scales and negative weights for the PF, RP, BP, and $\mathrm{GH}$ scales. Three scales (PF, RP, and BP) correlate most highly with the physical component and contribute most to the scoring of the PCS. The MCS correlates most highly with the $\mathrm{MH}, \mathrm{RE}$, and SF scales, which also contribute most to the scoring of the MCS measure. Three of the scales (VT, GH, and SF) have noteworthy correlations with both components. The SF-36 was constructed for self-administration by persons 14 years of age and 
older, and for administration by a trained interviewer in person or by telephone [26].

Adherence was assessed using the Validated Hemophilia Regimen Treatment Adherence Scale (VERITAS)-Pro [27] and VERITAS-PRN [28] for prophylactic and on-demand (i.e., episodic) participants, respectively. VERITAS scores range from 24 (most adherent) to 120 (least adherent). As an experimental measure, we also combined VERITAS-Pro and VERITAS-PRN responses into one category (VERITAS-combined) [25] to evaluate the relationship between adherence and HRQoL for both prophylactic and on-demand AYA PWH simultaneously. The cutoff for non-adherent prophylactic participants was a total VERITAS-Pro score $\geq 57$ as previously established [27]. The cutoff for non-adherent on-demand patients has not been previously described and was defined as those with VERITAS-PRN in the highest quartile of all responses. This value was chosen because the VERITAS-Pro cutoff was approximately the 75th percentile of all responses.

Chronic pain was measured using the revised Faces Pain Scale-Revised (FPS-R). The FPS-R is a visual scale composed of six faces illustrating an increasing level of pain intensity. Respondents were asked to choose the face that best describes the intensity of the chronic pain they experienced. In the IMPACT HRQoL survey, chronic pain was defined as 'pain that you have every day or almost every day, and that always or almost always seems to be there even when you are not having a bleed at that moment.' FPS-R scores range from 0 to 10 with the faces representing the lowest and highest levels of pain intensity coded as 0 and 10, respectively. The FPS-R is highly correlated with the visual analog scale $(\mathrm{r}=.93)$ and with the colored analog scale $(\mathrm{r}=.84)$, demonstrating strong validity. Reliability and validity of the FPS- $\mathrm{R}$ have been established for a broad age range, ranging from children as young as 4 years old to adults [29]. For purpose of analysis, chronic pain was dichotomized as high for those who reported their pain as 'moderate,' 'severe,' 'very severe, or 'worst pain possible' (i.e., FPS-R $\geq 4$ ) and low for 'mild pain' or 'no pain' (i.e., FPS-R $<4$ ).

Other self-reported data collected included information about participant age, gender, race, ethnicity, health insurance status/type, and the educational level of the participants' parents. Data were also collected about bleeding disorder type (hemophilia A or B, or VWD), whether or not the participant ever developed an inhibitor to treatment, and bleeding disorder severity. For hemophilia A and B, severity was classified as mild, moderate, or severe corresponding to 6 to $50 \%, 1$ to $5 \%$, and $<1 \%$, respectively, of the normal amount of clotting factor. VWD was classified as mild, moderate, or severe corresponding to Type I (lower than normal levels of von Willebrand factor), Type II (lower than normal levels and improper functioning of von Willebrand factor), and Type III disease (absence of von Willebrand factor in the blood).

\section{Statistical analysis}

Because of their skewed nature, descriptive statistics and univariate relationships were assessed by tabulating median PCS and MCS SF-36 scores with interquartile ranges (IQRs) by participant sociodemographic and clinical characteristics. Percentages were used to describe categorical variables and statistical association with SF36 scores was assessed using the non-parametric Wilcoxon rank sum test or Kruskal Wallis test (depending on degrees of freedom), as they do not assume a normal distribution of the residuals. As part of an exploratory analysis, we also examined univariate relationships between participant sociodemographic and clinical characteristics and each of the eight SF-36 subscales. Because this portion of the analysis was only exploratory, no adjustments for multiple comparisons were made.

The primary outcome variables of interest (SF-36 PCS and MCS scores) were largely skewed, thus multivariable, quantile regression models were used to assess factors associated with physical and emotional SF-36 subscales. Factors assessed for their relationship with HRQoL included: age, gender, race and ethnicity, parent's education level, bleeding disorder type and severity, history of inhibitor development, level of chronic pain, clotting-factor treatment adherence, and treatment regimen type (on-demand vs prophylactic). Due to the large number of variables collected as part of the survey and because of the small sample size inherent in rare disease research, in addition to the fully adjusted models, final parsimonious models were constructed. In the final parsimonious models, we decided, a priori, to include covariates in the model only if they (1) were statistically significant at a twotailed alpha level of .05, (2) changed the coefficient of another statistically significant model parameter by at least $10 \%$ to $15 \%$ (i.e., confounded) [30], or (3) improved the precision of another statistically significant parameter already in the model. All statistical analyses were performed using SAS 9.2 (SAS Institute, Inc; Cary, NC). All $p$-values were calculated using twosided tests.

\section{Results}

Respondent characteristics are summarized in Table 1. Overall, 108 AYAs with hemophilia A, hemophilia B, and VWD participated; half of the participants were aged 13 to 17 years, and the majority were male (83\%). Male participants were more likely to have hemophilia 
Table 1 Respondent characteristics $(n=108)$

\begin{tabular}{|c|c|c|}
\hline Characteristic & Number & Percent \\
\hline \multicolumn{3}{|l|}{ Age, years } \\
\hline $13-17$ & 54 & 50 \\
\hline $18-25$ & 54 & 50 \\
\hline \multicolumn{3}{|l|}{ Gender } \\
\hline Male & 90 & 83 \\
\hline Female & 18 & 17 \\
\hline \multicolumn{3}{|l|}{ Race } \\
\hline White & 86 & 80 \\
\hline Non-white ${ }^{a}$ & 22 & 20 \\
\hline \multicolumn{3}{|l|}{ Ethnicity } \\
\hline Hispanic & 12 & 11 \\
\hline Non-Hispanic & 96 & 89 \\
\hline \multicolumn{3}{|l|}{ Health insurance ${ }^{b}$} \\
\hline Medicaid or VA onlyc & 34 & 32 \\
\hline Commercial only & 46 & 43 \\
\hline Both & 8 & 7 \\
\hline Insured - type unknown & 12 & 11 \\
\hline Uninsured & 6 & 6 \\
\hline \multicolumn{3}{|l|}{ Mother's education level } \\
\hline Bachelor's degree or higher & 38 & 35 \\
\hline Less than Bachelor's degree & 70 & 65 \\
\hline \multicolumn{3}{|l|}{ Father's education level } \\
\hline Bachelor's degree or higher & 31 & 29 \\
\hline Less than Bachelor's degree & 71 & 71 \\
\hline \multicolumn{3}{|l|}{ Bleeding disorder } \\
\hline Hemophilia A & 85 & 79 \\
\hline Hemophilia B & 5 & 7 \\
\hline von Willebrand disease & 15 & 14 \\
\hline \multicolumn{3}{|l|}{ Severity } \\
\hline Mild & 22 & 20 \\
\hline Moderate & 9 & 8 \\
\hline Severe & 77 & 71 \\
\hline \multicolumn{3}{|l|}{ Inhibitor development } \\
\hline Ever & 38 & 35 \\
\hline Never & 70 & 65 \\
\hline \multicolumn{3}{|l|}{ Treatment regimen } \\
\hline On-demand & 35 & 32 \\
\hline Prophylaxis & 73 & 68 \\
\hline \multicolumn{3}{|l|}{ Chronic pain $^{d}$} \\
\hline None to mild & 74 & 69 \\
\hline Moderate to severe & 34 & 31 \\
\hline
\end{tabular}

Table 1 Respondent characteristics ( $n=108)$ (Continued)

Clotting factor adherence ${ }^{e}$
Adherent
Non-adherent
aMost (73\%) of non-white respondents were black or African American, 14\%
were mixed race, 9\% were Asian, and 5\% were American Indian or
Alaskan Native
b $\mathrm{n}=78$, two respondents answered 'Don't know' as to whether or not they
had health insurance, and were not included
'Only two respondents had VA only insurance, the others had Medicaid only
'Chronic pain was measured using the revised Faces Pain Scale (FPS-R) and
was dichotomized as FPS-R $<4$ (i.e., 'mild' or 'no pain) and FPS-R $\geq 4$
(ie, 'moderate' to 'worst pain possible')
eAdherence was assessed using the Validated Hemophilia Regimen Treatment
Adherence Scale (VERITAS)-Pro and VERITAS-PRN for prophylactic and on-demand
participants, respectively. The cutoff for non-adherent prophylactic participants
was a total VERITAS-Pro score $\geq 57$ as established in previously by Duncan and
colleagues [25]. This value was chosen because the VERITAS-Pro cutoff was
approximately the 75th percentile of all responses

vs VWD (96 vs 4\%), while the opposite was true for female participants (39 vs 61\%). The majority (94\%) of respondents had some type of health insurance.

Median PCS and MCS were 81.3 (IQR: 61.1-93.1; range: 12.9-100) and 75.5 (IQR: 60.0-84.3.1; range: 27.1-100), respectively (Figs. 1 and 2). Mean values for PCS, MCS, and the eight multi-item subscales were generally lower than the median (with the exception of VT and SF) due to low outlying values (Fig. 3). At the univariate level, young adults (vs adolescents), non-whites, those who reported ever developing an inhibitor, and those who reported moderate to severe (vs none to mild) chronic pain had statistically significantly lower PCS scores (Table 2). Young adults (vs adolescents), those who reported moderate to severe (vs none to mild) chronic pain, and those who were non-adherent to prescribed clotting-factor treatment regimens had statistically significantly lower MCS scores (Table 2). Univariate level differences for PCS, MCS, and the

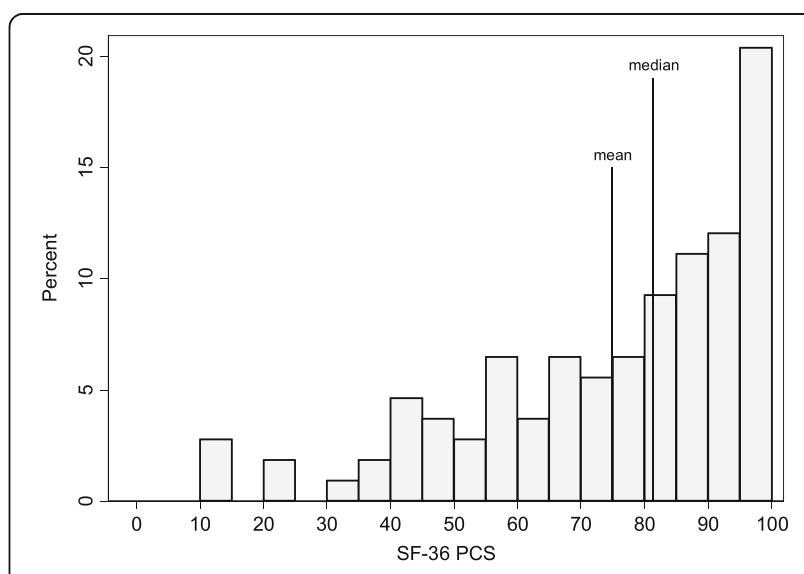

Fig. 1 Distribution of SF-36 Physical Component Summary scores $(n=108)$. SF-36, 36-Item Short Form Health Survey; PCS, Physical Component Summary 


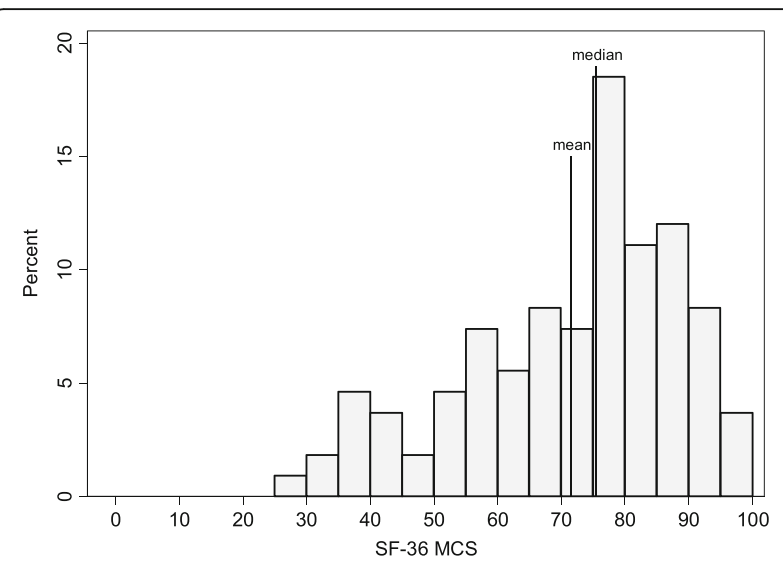

Fig. 2 Distribution of SF-36 Mental Component Summary scores ( $n=108)$. SF-36, 36-Item Short Form Health Survey; MCS, Mental Component Summary

eight multi-item subscales by respondent characteristic are shown in the supplement (Figs 4 and 5 for median and mean values, respectively).

Final quantile regression models suggested that, compared with men, median PCS for women was 13.1 (95\% CI: 2.4, 23.8; $p=0.02$ ) points lower. Ever developing an inhibitor (vs never) was significantly associated with a 13.1 (95\% CI: 4.7, 21.5; $p<0.01)$ point reduction in PCS. Prophylactic infusers (vs ondemand) had a 10.0 (95\% CI: 0.7, 19.3; $p=0.04$ ) point increase in median MCS. Compared with those with no or mild chronic pain, those with moderate to severe chronic pain had a 25.5 (95\% CI: 17.2, 33.8; $p<0.001)$ and 10.0 (95\% CI: $0.8,19.2 ; p=0.03$ ) point reduction in

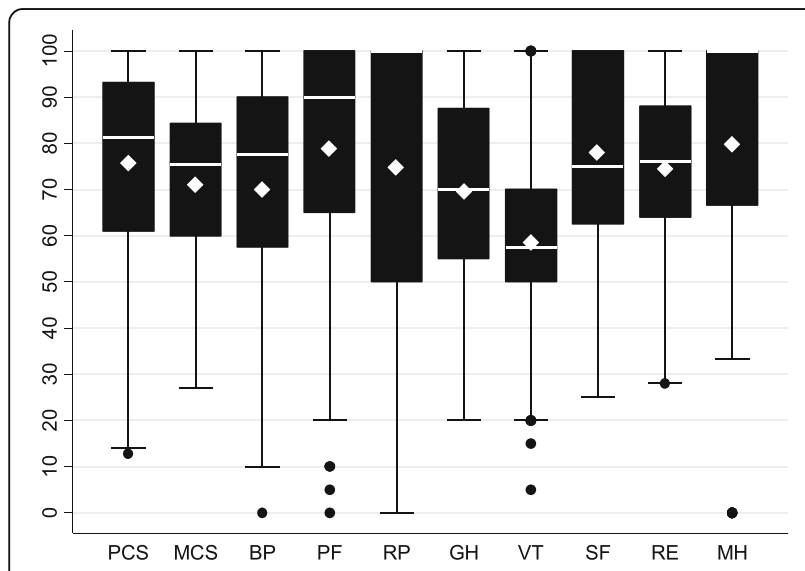

Fig. 3 Box plots for SF-36 component and subscale scores $(n=108)$. The solid white line represents median value, and the white diamond represents the mean value. PCS, Physical Component Summary score; MCS, Mental Component Summary; BP, bodily pain; PF, physical function; RP, role limitations due to physical health problems; $\mathrm{GH}$, general health; VT, vitality; SF, social functioning; $\mathrm{RE}$, role limitations due to emotional problems; $\mathrm{MH}$, emotional well-being/mental health median PCS and MCS, respectively. Tables 3 and 4 show final quantile regression models modeling PCS and MCS scores, respectively.

\section{Discussion}

Evaluating HRQoL will be required to determine the effectiveness of care strategies and to measure the impact of living with a bleeding disorder over time. To our knowledge, limited data are available regarding the HRQoL of AYAs with hemophilia. In contrast to previous surveys, such as Hemophilia Experiences, Results and Opportunities (HERO), that assessed psychosocial issues in PWH [31-33], we assessed adherence using the validated VERITAS scale and used the SF-36 (vs the EuroQoL-5 dimensions [EQ-5D]) to assess HRQoL. The SF-36 assesses domains such as vitality and social functioning, which are not included in the EQ-5D [34]. Additionally, all patients in our study self-reported for assessments of QoL, whereas parents responded on behalf of children younger than 18 years in HERO [32]. Consistent with previous research in PWH among other age groups [35], AYA PWH or VWD who reported they had ever developed an inhibitor and those who reported moderate-to-severe chronic pain also reported worse physical HRQoL in this study. Previous reports suggest this finding is likely explained by the fact that most PWH report that pain interferes with daily activities and has a negative impact on their work. In addition to physical limitations in daily activity and work, prior research has suggested an emotional impact of chronic pain on quality of life as well [36, 37]. Witkop et al. demonstrated that one third of PWH report that pain impairs the ability to form close relationships and that nearly half of young adults with a bleeding disorder report being diagnosed with anxiety or depression [37]. It has been suggested that, in addition to emphasizing prophylaxis, interventions to promote acceptance of pain and to reduce negative thoughts about pain should be utilized when approaching PWH [15].

Adolescents had higher PCS and MCS scores compared to young adults, which may be related to more frequent disease-related bleeding and greater prevalence of chronic pain as individuals managing a bleeding disorder transition from adolescence to young adulthood. Previous research has underscored that adherence to treatment regimens declines with age. Diligent factor administration by parents in younger years often gives way to less treatment regimen fidelity during young adulthood where patients often begin to assume primary responsibility for their bleeding disorder care [22, 23, 38, 39]. Further, as children transition into young adulthood, their activity level often intensifies, which could heighten the risk of bleeding. 
Table 2 Median (IQR) SF-36 Physical and Mental Component Summary scores by respondent characteristic $(n=108)$

\begin{tabular}{|c|c|c|c|c|}
\hline Characteristic & SF-36 PCS & $p$-value & SF-36 MCS & $p$-value \\
\hline Age & & 0.03 & & 0.04 \\
\hline $13-17$ & $86.3(64.8,95.7)$ & & $79.5(64.6,85.7)$ & \\
\hline $18-25$ & $75.1(59.0,87.1)$ & & $73.4(58.6,78.9)$ & \\
\hline Gender & & 0.12 & & 0.10 \\
\hline Male & $83.0(59.0,94.5)$ & & $76.8(59.6,85.7)$ & \\
\hline Female & $78.9(64.8,86.0)$ & & $71.4(64.6,76.4)$ & \\
\hline Race & & 0.02 & & 0.94 \\
\hline White & $83.6(65.7,94.3)$ & & $75.9(59.6,84.3)$ & \\
\hline Non-white $^{a}$ & $62.6(48.3,84.8)$ & & $74.3(64.6,85.7)$ & \\
\hline Ethnicity & & 0.18 & & 0.73 \\
\hline Hispanic & $71.9(59.3,81.5)$ & & $77.0(67.0,82.1)$ & \\
\hline Non-Hispanic & $83.0(61.1,94.2)$ & & $75.2(59.6,84.8)$ & \\
\hline Health insurance ${ }^{b}$ & & 0.13 & & 0.21 \\
\hline Medicaid or VA only ${ }^{c}$ & $79.4(56.0,86.4)$ & & $75.9(64.6,85.7)$ & \\
\hline Commercial only & $86.2(65.0,95.7)$ & & $78.8(65.0,84.3)$ & \\
\hline Both & $71.0(60.8,77.7)$ & & $72.9(59.3,81.3)$ & \\
\hline Insured, type unknown & $83.7(69.6,91.8)$ & & $64.3(54.6,86.3)$ & \\
\hline Uninsured & $63.0(41.4,90.5)$ & & $61.8(42.9,69 / 3)$ & \\
\hline Mother's education level & & 0.19 & & 0.71 \\
\hline Bachelor's degree or higher & $86.1(65.7,95.5)$ & & $75.7(60.4,85.4)$ & \\
\hline Less than Bachelor's degree & $80.1(58.1,91.0)$ & & $75.5(59.6,84.3)$ & \\
\hline Father's education level & & 0.10 & & 0.46 \\
\hline Bachelor's degree or higher & $88.3(67.4,95.5)$ & & $76.4(58.6,87.1)$ & \\
\hline Less than Bachelor's degree & $79.8(58.1,91.0)$ & & $75.4(60.4,82.9)$ & \\
\hline Bleeding disorder & & 0.78 & & 0.34 \\
\hline Hemophilia A & $81.2(58.1,94.5)$ & & $76.4(59.3,85.7)$ & \\
\hline Hemophilia B & $81.7(73.2,88.5)$ & & $76.1(65.0,81.8)$ & \\
\hline von Willebrand & $80.5(64.8,86.2)$ & & $70.0(64.6,76.4)$ & \\
\hline Severity & & 0.98 & & 0.37 \\
\hline Mild & $81.6(65.0,91.7)$ & & $73.2(61.8,78.6)$ & \\
\hline Moderate & $86.0(64.0,88.6)$ & & $75.4(60.4,87.1)$ & \\
\hline Severe & $79.8(58.1,94.3)$ & & $76.4(59.6,85.7)$ & \\
\hline Inhibitor development & & $<0.01$ & & 0.16 \\
\hline Ever & $69.0(45.0,85.2)$ & & $73.4(56.8,83.9)$ & \\
\hline Never & $86.1(67.9,94.5)$ & & $78.2(64.6,86.4)$ & \\
\hline Treatment regimen & & 0.99 & & 0.13 \\
\hline On-demand & $81.9(66.2,91.7)$ & & $72.9(59.3,78.9)$ & \\
\hline Prophylaxis & $81.2(58.1,94.5)$ & & $77.1(60.4,85.7)$ & \\
\hline Chronic pain ${ }^{d}$ & & $<0.0001$ & & 0.01 \\
\hline None to mild & $87.1(75.5,95.5)$ & & $77.1(64.6,86.8)$ & \\
\hline Moderate to severe & $59.0(45.0,74.5)$ & & $72.9(56.8,78.8)$ & \\
\hline
\end{tabular}


Table 2 Median (IQR) SF-36 Physical and Mental Component Summary scores by respondent characteristic $(n=108)($ Continued)

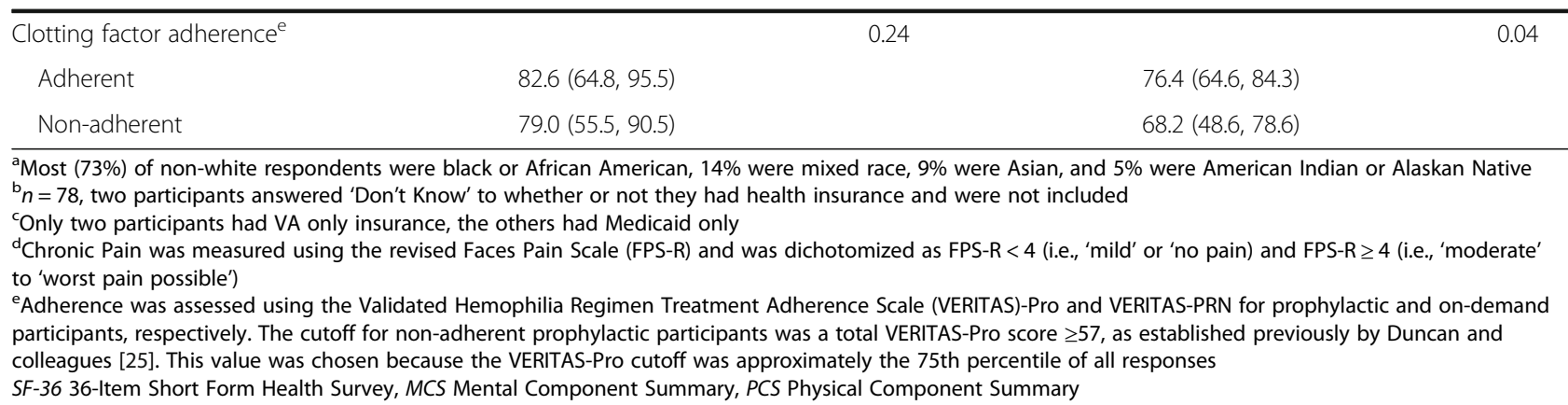

AYA females with a bleeding disorder reported lower physical HRQoL when compared with AYA men in this study, even after adjustment for other sociodemographic and clinical factors. Reasons for this are not clear. Previous work would suggest the opposite might be true given that, compared with AYA males, female AYA were more likely to have VWD (vs hemophilia) and that VWD, generally speaking, has a lower overall burden of disease than does hemophilia and is less commonly associated with the cumulative arthropathic burden in adulthood often seen in PWH [40]. However, approximately one in four patients with VWD experience joint bleeds, which can have a negative effect on physical quality of life, with the majority of first joint bleeds occurring before age 16 [40]. In addition, previous research has suggested that physical quality of life in female VWD patients may be affected by menorrhagia, dysmenorrhagia, or pregnancy-related bleeding, which could lead to anemia, fatigue, and pain [41-43]. Thus, in AYA, it is possible that acute bleeding and dysmenorrhea often associated with female VWD patients have a more immediate deleterious effect on HRQoL than do the long-term effects (e.g., arthropathy and hepatitis C) associated with hemophilia which may have

Table 3 Quantile (median) regression model estimating median SF-36 Physical Component Summary scores, $2012(n=108)$

\begin{tabular}{lll}
\hline Characteristic & Coefficient $(95 \% \mathrm{Cl})$ & $p$-value \\
\hline Gender & & 0.02 \\
Female & reference & \\
Male & & $<0.1(-23.8,-2.4)$ \\
Inhibitor development & $-13.1(-21.5,-4.7)$ & \\
Ever & reference & $<0.001$ \\
Never & & \\
Chronic pain & \\
$\quad$ Moderate to severe & $-25.5(-33.8,-17.2)$ & \\
None to mild & reference & \\
\hline
\end{tabular}

${ }^{a}$ Chronic pain was measured using the revised Faces Pain Scale (FPS-R) and was dichotomized as FPS-R $<4$ (i.e., 'mild' or 'no pain) and FPS-R $\geq 4$ (i.e., 'moderate' to 'worst pain possible') not yet fully manifested. This interaction between age, sex, bleeding disorder type, and HRQoL should be more thoroughly evaluated with future research.

AYA patients who infused clotting factor prophylactically reported significantly better mental HRQoL (MCS) compared with those who infused using ondemand regimens. However, differences in physical HRQoL (PCS) were not observed in our study, which is a surprising finding given that prophylaxis is associated with fewer joint bleeds and less pain [7], and a recent review found that joint status was associated with both social and emotional well-being [44]. One possible explanation for the findings in our study is that physical sequelae of the disease have not yet manifested in AYAs, a hypothesis also set forth by Poon et al. [20]. However, prophylactic treatment may provide mental assurance to patients that their disease is well controlled, thereby leading to improved mental HRQoL. Future studies should attempt to tease out the influence of prophylaxis, disease type and severity, and comorbid illness on HRQoL.

This study has limitations. Primarily, data are crosssectional, thus causal inference cannot be made. Specifically, although study results support that several factors (e.g., chronic pain, inhibitor development, clotting-factor regimen type) are related to HRQoL,

Table 4 Quantile (median) regression model estimating median SF-36 Mental Component Summary scores, $2012(n=108)^{a}$

\begin{tabular}{lll}
\hline Characteristic & Coefficient $(95 \% \mathrm{Cl})$ & $p$-value \\
\hline $\begin{array}{l}\text { Treatment regimen } \\
\text { Prophylaxis }\end{array}$ & 0.04 \\
$\begin{array}{l}\text { On-demand } \\
\text { Chronic pain }\end{array}$ & \\
Modererence & \\
None to mild & $-10.0(-19.2,-0.8)$ & 0.03 \\
& reference & \\
\hline
\end{tabular}

aEthnicity (Hispanic vs non-Hispanic) and history of inhibitor development (ever vs never) were also included in the model, though not statistically significant, because they increased the precision of the estimates ${ }^{\mathrm{b}}$ Chronic pain was measured using the revised Faces Pain Scale (FPS-R) and was dichotomized as FPS-R $<4$ (i.e., 'mild' or 'no pain) and FPS-R $\geq 4$ (i.e., 'moderate' to 'worst pain possible')

Cl confidence interval 
the directionality of this relationship cannot be confirmed. That is, though we modeled how these factors affect HRQoL, it is also possible that HRQoL instead affects a patient's willingness and ability to adequately manage his or her bleeding disorder. This cannot be teased out in a cross-sectional study and should be examined in the future with prospective studies. A second limitation is that all data are self-reported. As such, information about blood disorder type and severity, health insurance coverage, and other demographic, clinical, and behavioral factors are not confirmed by medical record review or administrative claims data. However, by obtaining data through selfreport, this study was able to collect important, reliable, and valid patient-report outcomes (PRO) data about adherence, chronic pain, and HRQoL. Patientreported data about pain and HRQoL are considered the 'gold standard', and the VERITAS scales are the only validated measures of adherence to clottingfactor treatment developed to date.

Another limitation is that the VERITAS-combined score that we reported, though statistically comprised of two validated instruments, is an experimental, nonvalidated measure that has only been previously reported once [25]. Most participants (68\%), however, treated prophylactically, and VERITAS-Pro scores, which have been previously validated, confirmed the results of the experimental, combined score. Additionally, the SF36 , used in this study to assess HRQoL, is only validated in persons aged 14 years and older [26], whereas a small percentage of patients in our study were aged 13 years $(\mathrm{n}=9 / 108,8.3 \%)$. Further, no comparisons were made between SF-36 results in study respondents versus matched normal controls or persons living with other chronic diseases. Finally, AYA PWH and VWD were primarily recruited from large national or regional hemophilia meetings. Thus, our convenience sample of AYA PWH and VWD may not adequately represent the broader AYA PWH and VWD population who do not typically attend these meetings.

\section{Conclusions}

Efforts should be made to prevent and manage chronic pain, which was strongly related to both physical and mental HRQoL, in the AYA PWH and VWD populations. Previous research has suggested that better adherence to prescribed treatment regimens is associated with less chronic pain [25]. Future research should explore (1) why women had lower physical HRQoL scores-even after adjustment for other sociodemographic and clinical factors, and (2) how prophylaxis may improve overall mental/emotional HRQoL in the AYA PWH and VWD populations.

\section{Abbreviations}

AYA: Adolescent and young adult; BP: Bodily pain; Cl: Confidence interval; EQ-5D: EuroQoL-5 dimensions; FPS-R: Faces Pain Scale-Revised; GH: General health; HRQoL: Health-related quality of life; IQR: Interquartile ranges; MCS: Mental Component Score; MH: Emotional well-being/mental health; PCS: Physical Component Score; PF: Physical function; PWH: Persons with hemophilia; RE: Role limitations due to emotional problems; RP: Role limitations due to physical health problems; SF: Social functioning; SF-36: 36-Item Short Form Health Survey; VT: Vitality; WWD: von Willebrand disease

\section{Acknowledgments}

Editorial support for formatting the manuscript as per journal requirements was provided by Teri ONeill at Peloton Advantage, and was funded by Pfizer Inc. This study was sponsored by Pfizer Inc.

\section{Funding}

This study was sponsored by Pfizer Inc. This study and manuscript were funded by Pfizer Inc. Pfizer employees were involved in the study design, the collection, analysis, and interpretation of data, the review of the manuscript, and the decision to submit for publication.

\section{Availability of data and materials}

The data that support the findings of this study are available from Pfizer Inc but restrictions apply to the availability of these data, which were used under license for the current study, and so are not publicly available. Data are, however, available from the authors upon reasonable request and with the permission of Pfizer Inc.

\section{Authors' contributions}

JMM, JEM, BT, AL and MLW contributed to the study design. JMM, JEM, AL and MLW participated in the collection and assembly of data. JMM, JEM, $\mathrm{BT}, \mathrm{AL}$ and MLW participated in data analysis and interpretation. All authors participated in manuscript preparation, critically reviewed and provided revisions to the manuscript, and granted final approval of the manuscript for submission.

\section{Competing interests}

Drs. McLaughlin, Anderson, and Tortella are employees and shareholders of Pfizer Inc. Dr. Witkop and Mr. Munn have no conflicts of interest to report. At the time of this research, Mrs. Lambing had no conflicts of interest to report.

\section{Consent for publication}

Not applicable.

\section{Ethics approval and consent to participate}

Informed consent was obtained from all individual participants included in the study. The study was approved by the Munson Medical Center (Traverse City, MI) institutional review board prior to data collection. All authors have consented to the publication of this manuscript.

\section{Publisher's note}

Springer Nature remains neutral with regard to jurisdictional claims in published maps and institutional affiliations.

\section{Author details}

'Pfizer Inc, Global Innovative Pharma, 500 Arcola Rd, Collegeville, PA 19426, USA. ${ }^{2}$ University of Michigan Hemophilia Treatment Center, 1500 E. Medical Center Drive, Ann Arbor, MI, USA. ${ }^{3}$ Henry Ford Hemophilia \& Thrombosis Treatment Center, 2799 W Grand Blvd, Detroit, MI, USA. ${ }^{4}$ Northern Regional Bleeding Disorder Center, 1105 Sixth St, Traverse City, MI, USA. ${ }^{5}$ Currently: Bayer HealthCare, Whippany, NJ, USA.

Received: 3 November 2016 Accepted: 29 March 2017

Published online: 07 April 2017

\section{References}

1. Escobar MA. Advances in the treatment of inherited coagulation disorders. Haemophilia. 2013;19(5):648-59.

2. Kaufman RJ, Powell JS. Molecular approaches for improved clotting factors for hemophilia. Blood. 2013;122(22):3568-74. 
3. Lusher JM, Arkin S, Abildgaard CF, Schwartz RS. Recombinant factor VIII for the treatment of previously untreated patients with hemophilia a. Safety, efficacy, and development of inhibitors. Kogenate previously untreated patient study group. N Engl J Med. 1993;328(7):453-9.

4. Murphy SL, High KA. Gene therapy for haemophilia. Br J Haematol. 2008; 140(5):479-87.

5. Franchini M, Mengoli C, Lippi G, Targher G, Montagnana M, Salvagno GL, et al. Immune tolerance with rituximab in congenital haemophilia with inhibitors: a systematic literature review based on individual patients' analysis. Haemophilia. 2008;14(5):903-12.

6. Gringeri A, Lundin B, von Mackensen S, Mantovani L, Mannucci PM, Group ES. A randomized clinical trial of prophylaxis in children with hemophilia A (the ESPRIT Study). J Thromb Haemost. 2011;9(4):700-10.

7. Manco-Johnson MJ, Abshire TC, Shapiro AD, Riske B, Hacker MR, Kilcoyne R, et al. Prophylaxis versus episodic treatment to prevent joint disease in boys with severe hemophilia. N Engl J Med. 2007;357(6):535-44.

8. Nilsson IM, Berntorp E, Lofqvist T, Pettersson H. Twenty-five years' experience of prophylactic treatment in severe haemophilia a and B. J Intern Med. 1992;232(1):25-32

9. Evatt BL. The natural evolution of haemophilia care: developing and sustaining comprehensive care globally. Haemophilia. 2006;12 suppl 3: $13-21$.

10. Hoots WK. Comprehensive care for hemophilia and related inherited bleeding disorders: why it matters. Curr Hematol Rep. 2003;2(5):395-401.

11. Smith PS, Levine PH. The benefits of comprehensive care of hemophilia: a five-year study of outcomes. Am J Public Health. 1984;74(6):616-7.

12. Soucie JM, Nuss R, Evatt B, Abdelhak A, Cowan L, Hill H, et al. Mortality among males with hemophilia: relations with source of medical care. The hemophilia surveillance system project investigators. Blood. 2000;96(2): 437-42.

13. Konkle BA. Clinical challenges within the aging hemophilia population. Thromb Res. 2011;127 Suppl 1:S10-3.

14. Scalone L, Mantovani LG, Mannucci PM, Gringeri A, Investigators CS. Quality of life is associated to the orthopaedic status in haemophilic patients with inhibitors. Haemophilia. 2006;12(2):154-62.

15. Elander J, Morris J, Robinson G. Pain coping and acceptance as longitudinal predictors of health-related quality of life among people with haemophiliarelated joint pain. Eur J Pain. 2013;17(6):929-38.

16. Gringeri A, Leissinger C, Cortesi PA, Jo H, Fusco F, Riva S, et al. Healthrelated quality of life in patients with haemophilia and inhibitors on prophylaxis with anti-inhibitor complex concentrate: results from the Pro-FEIBA study. Haemophilia. 2013;19(5):736-43.

17. Khair K. Supporting adherence and improving quality of life in haemophilia care. Br J Nurs. 2013;22(12):692

18. Kim SY, Kim SW, Kim JM, Shin IS, Baek HJ, Lee HS, et al. Impact of personality and depression on quality of life in patients with severe haemophilia in Korea. Haemophilia. 2013;19(5):e270-5.

19. Mondorf W, Kalnins W, Klamroth R. Patient-reported outcomes of 182 adults with severe haemophilia in Germany comparing prophylactic vs. On-demand replacement therapy. Haemophilia. 2013;19(4):558-63.

20. Poon JL, Zhou ZY, Doctor JN, Wu J, Ullman MM, Ross C, et al. Quality of life in haemophilia a: hemophilia utilization group study Va (HUGS-Va). Haemophilia. 2012;18(5):699-707.

21. Witkop M, Lambing A, Divine G, Kachalsky E, Rushlow D, Dinnen J. A national study of pain in the bleeding disorders community: a description of haemophilia pain. Haemophilia. 2012;18(3):e115-9.

22. Schrijvers LH, Uitslager N, Schuurmans MJ, Fischer K. Barriers and motivators of adherence to prophylactic treatment in haemophilia: a systematic review. Haemophilia. 2013;19(3):355-61.

23. Lindvall K, Colstrup L, Wollter IM, Klemenz G, Loogna K, Gronhaug S, et al. Compliance with treatment and understanding of own disease in patients with severe and moderate haemophilia. Haemophilia. 2006; 12(1):47-51.

24. McLaughlin JM, Lambing A, Witkop ML, Anderson TL, Munn J, Tortella B. Racial differences in chronic pain and quality of life among adolescents and young adults with moderate to severe hemophilia. J Racial Ethn Health Disparities. 2016;3(1):11-20

25. McLaughlin JM, Witkop ML, Lambing A, Anderson TL, Munn J, Tortella B. Better adherence to prescribed treatment regimen is related to less chronic pain among adolescents and young adults with moderate or severe haemophilia. Haemophilia. 2014;20(4):506-12.
26. Ware Jr JE, Sherbourne CD. The MOS 36-item short-form health survey (SF-36). I. Conceptual framework and item selection. Med Care. 1992; 30(6):473-83

27. Duncan N, Kronenberger W, Roberson C, Shapiro A. VERITAS-Pro: a new measure of adherence to prophylactic regimens in haemophilia. Haemophilia. 2010;16(2):247-55.

28. Duncan NA, Kronenberger WG, Roberson CP, Shapiro AD. VERITAS-PRN: a new measure of adherence to episodic treatment regimens in haemophilia. Haemophilia. 2010;16(1):47-53.

29. Hicks CL, von Baeyer CL, Spafford PA, van Korlaar I, Goodenough B. The faces pain scale-revised: toward a common metric in pediatric pain measurement. Pain. 2001;93(2):173-83.

30. Mickey RM, Greenland S. The impact of confounder selection criteria on effect estimation. Am J Epidemiol. 1989;129(1):125-37.

31. Cassis FR, Buzzi A, Forsyth A, Gregory M, Nugent D, Garrido C, et al. Haemophilia experiences, results and opportunities (HERO) study: influence of haemophilia on interpersonal relationships as reported by adults with haemophilia and parents of children with haemophilia. Haemophilia. 2014; 20(4):e287-95.

32. Forsyth AL, Gregory M, Nugent D, Garrido C, Pilgaard T, Cooper DL, et al. Haemophilia experiences, results and opportunities (HERO) study: survey methodology and population demographics. Haemophilia. 2014;20(1): 44-51.

33. Nugent D, Kalnins W, Querol F, Gregory M, Pilgaard T, Cooper DL, et al. Haemophilia experiences, results and opportunities (HERO) study: treatment-related characteristics of the population. Haemophilia. 2015; 21(1):e26-38

34. Joore M, Brunenberg D, Nelemans P, Wouters E, Kuijpers P, Honig A, et al. The impact of differences in EQ-5D and SF-6D utility scores on the acceptability of cost-utility ratios: results across five trial-based cost-utility studies. Value Health. 2010;13(2):222-9.

35. Fischer K, van der Bom JG, van den Berg HM. Health-related quality of life as outcome parameter in haemophilia treatment. Haemophilia. 2003;9 supp 1:75-81. discussion 2.

36. Elander J, Robinson G, Mitchell K, Morris J. An assessment of the relative influence of pain coping, negative thoughts about pain, and pain acceptance on health-related quality of life among people with hemophilia. Pain. 2009;145(1-2):169-75

37. Witkop M, Guelcher C, Forsyth A, Hawk S, Curtis R, Kelley L, et al. Treatment outcomes, quality of life, and impact of hemophilia on young adults (aged 18-30 years) with hemophilia. Am J Hematol. 2015;90 Suppl 2:S3-10.

38. du Treil S, Rice J, Leissinger CA. Quantifying adherence to treatment and its relationship to quality of life in a well-characterized haemophilia population. Haemophilia. 2007:13(5):493-501.

39. Geraghty S, Dunkley T, Harrington C, Lindvall K, Maahs J, Sek J. Practice patterns in haemophilia A therapy - global progress towards optimal care. Haemophilia. 2006;12(1):75-81.

40. van Galen KP, Sanders YV, Vojinovic U, Eikenboom J, Cnossen MH, Schutgens RE, et al. Joint bleeds in von Willebrand disease patients have significant impact on quality of life and joint integrity: a cross-sectional study. Haemophilia. 2015;21(3):e185-92.

41. de Wee EM, Mauser-Bunschoten EP, Van Der Bom JG, Degenaar-Dujardin ME, Eikenboom HC, Fijnvandraat K, et al. Health-related quality of life among adult patients with moderate and severe von Willebrand disease. J Thromb Haemost. 2010:8(7):1492-9.

42. Kouides PA. Menorrhagia from a haematologist's point of view. Part I: initial evaluation. Haemophilia. 2002:8(3):330-8.

43. Kouides PA, Phatak PD, Burkart P, Braggins C, Cox C, Bernstein Z, et al Gynaecological and obstetrical morbidity in women with type I von Willebrand disease: results of a patient survey. Haemophilia. 2000;6(6):643-8.

44. Elander J. A review of evidence about behavioural and psychological aspects of chronic joint pain among people with haemophilia. Haemophilia. 2014:20(2):168-75. 